\title{
A platform for screening abiotic/biotic interactions using indicator displacement assays
}

Monika Michaelis, ${ }^{1,2 \ddagger}$ Aneeqa Fayyaz, ${ }^{1 \ddagger}$ Mithun Parambath, ${ }^{1 \ddagger}$ Susan Koeppen, ${ }^{2}$ Lucio Colombi

Ciacchi, ${ }^{2}$ Quentin S. Hanley, ${ }^{3}$ Carole C. Perry ${ }^{* 1}$

${ }^{1}$ Biomolecular and Materials Interface Research Group, School of Science and Technology, Nottingham Trent University, Clifton Lane, Nottingham, UK NG11 8NS; ${ }^{2}$ University of Bremen, Hybrid Materials Interfaces Group, Faculty of Production Engineering, Bremen Center for Computational Material Science (BCCMS), Center for Environmental Research and Sustainable Technology (UFT) and MAPEX Centre for Materials and Processes, D-28359 Bremen; ${ }^{3}$ The Analytical Chemistry Research Group. School of Science and Technology, Nottingham Trent University, Clifton Lane, Nottingham, UK NG11 8NS

KEYWORDS: Biomaterials; interface; abiotic/biotic interactions; indicator displacement assay; O-IDA; fluorescence; binding affinity; free energy of adsorption; $\mathrm{EC}_{50}$; $\mathrm{IC}_{50}$; Silica; ZnO; drugs; amino acids; polyamine. 


\begin{abstract}
This paper describes novel adaptations of optically sectioned planar format assays to screen compounds for their affinities to materials surfaces. The novel platform, which we name Optical sectioned Indicator Displacement Assays (O-IDA), makes use of displaceable dyes in a format adaptable to high-throughput multi-well plate technologies. We describe two approaches; the first being where the dye exhibits fluorescence in both the surface bound and unbound state and the second, where fluorescence is lost upon displacement of the dye from the surface. Half maximal inhibitory concentration ( $\left.\mathrm{IC}_{50}\right)$, binding affinity $\left(K_{i}\right)$, and binding free energy $\left(\Delta G_{a d s}\right)$ values can be extracted from the raw data. Representative biomolecules were tested for interactions with silica in aqueous environment and $\mathrm{ZnO}(0001)-\mathrm{Zn}$ and (10-10) facets in a non-aqueous environment. We provide the first experimental values for both the binding of small molecules to silica and the facetdependent ZnO binding affinity of key amino acids associated with ZnO-specific oligopeptides. The specific data will be invaluable to those studying interactions at interfaces both experimentally and computationally. O-IDA provides a general framework for the high-throughput screening of molecules binding to materials surfaces, which has important applications in drug delivery, (bio-) catalysis, biosensing and biomaterials engineering.
\end{abstract}

* Corresponding author: carole.perry@ntu.ac.uk 


\section{Introduction}

Interactions at the abiotic/biotic interface are of fundamental interest in medical devices, drug delivery and catalysis and have wide applications ranging from polypeptide adsorption, ${ }^{1-4}$ cell adhesion, ${ }^{5,6}$ biomaterials, ${ }^{7-10}$ and biosensors. ${ }^{11,12}$ Common methods for the experimental quantification of interactions at materials' surfaces include quartz crystal microbalance (QCM), ${ }^{13}$ surface plasmon resonance (SPR) ${ }^{14}$ or single molecule force spectroscopy (SMFS) ${ }^{15}$ These techniques take an indirect measure of the interactions and heavily rely on the models applied during data analysis. Furthermore, the experimental procedure and the associated analysis can be very time consuming. A direct measurement of binding enthalpies can be achieved using isothermal titration calorimetry ${ }^{16}$ but the method is limited to stable suspensions.

Indicator displacement assays ${ }^{17-19}$ provide measurements of interactions based on the direct observation of competitive binding between a reference compound and the analyte of interest to a given interaction site. Although they are used in drug discovery and high throughput screening, to the best of our knowledge, they have not previously been applied to screening of molecules that bind to abiotic material surfaces. Adapting this approach into the field of abiotic/biotic interactions would represent an important advance and could enable comprehensive screening of interactions at the analyte/material interface. Screenable displacement assays in a planar format ${ }^{20}$ have been described for receptor ligand interaction studies and this approach can indeed be adapted to measure molecular interactions with abiotic/biotic surfaces, as we will prove in this paper.

We establish the novel approach using two representative functional oxides with important applications in nanotechnology and biomedicine. The first system is silica, for which knowledge of important binding interactions can lead to a deeper understanding of silica-based drug delivery 
systems, ${ }^{21,22}$ the synthesis of new materials for biomedical use ${ }^{23}$ and processes such as biosilicification ${ }^{24,25}$ in mineralizing organisms. ${ }^{26-28}$ Advancements in this research area will have direct implications in material science, medicine and bio-nanotechnology. For this system we selected representative amino acids, a small polyamine ${ }^{29}$ and a drug, ibuprofen, that is often used in model studies. ${ }^{30-32}$

As a second system, we chose zinc oxide which is widely used in the form of crystals and nanoparticles for applications ranging from (photo-) catalysis, ${ }^{33}$ mechanical actuators, ${ }^{34}$ piezoelectric and optoelectronic devices ${ }^{35}$ through to biomedical applications. ${ }^{36}$ This versatility is triggered by the unique properties of $\mathrm{ZnO}$, which is a semiconductor with a wide direct band gap and a high exciton binding energy. ${ }^{37}$ The morphology-dependent properties of $\mathrm{ZnO}$ can be altered using biomolecules, which lead to a large variety of shapes with small variations in the preparation conditions. Within this framework, the biomineralization of $\mathrm{ZnO}$ with amino acids is of special interest. ${ }^{38-43}$ For the selection of amino acids (H, C, S, L, A and P), we refer to prior studies where the interaction of binding peptides with specific $\mathrm{ZnO}$ crystal planes has been investigated. ${ }^{43,44}$ Further, histidine and cysteine are well known to bind $\mathrm{Zn}^{2+}$ ions in zinc finger proteins $\mathrm{s}^{45-48}$ and while histidine was found to be enriched in a phage display study for two common surfaces of $\mathrm{ZnO}$, namely the zinc-terminated (0001) and the oxygen-terminated (000-1) polar surfaces, ${ }^{44}$ cysteine is usually suppressed due to the impairment of phage infectivity. ${ }^{49,50}$

This paper describes an approach to measure abiotic-biotic interactions which involves the combination of optically sectioned planar format assays (OSPFA) using confocal scanning laser microscopy (CSLM) and an indicator displacement assay (IDA) for screening of compounds binding to abiotic surfaces. The goal is to enable high throughput screening of molecule libraries to identify compounds with high materials binding affinity. Although our examples are two 
functional oxides, silica and $\mathrm{ZnO}$, the approaches introduced here are general and could be applied to a wide range of materials.

\section{Materials and Methods}

Silica: Hydrophilic silica surfaces were produced following a previously described protocol, ${ }^{51-53}$ a brief description is provided in the SI. CSLM experiments were conducted using 96 microwell glass bottom black plates (Nunc 164588). To determine the binding constant of the PDMPO dye ((LysoSensor ${ }^{\mathrm{TM}}$ yellow/blue DND-160, $1 \mathrm{mM}$ in dimethyl sulfoxide), Life Technologies) to the silica surfaces, we measured the concentration-dependent adsorption behavior and the timedependent increase of the fluorescence intensity upon interaction with the substrate. To measure the binding of the compounds of interest to the silica surface the following protocol was adopted. $1 \mu \mathrm{M}$ PDMPO ((LysoSensorTM yellow/blue DND-160, $1 \mathrm{mM}$ in dimethyl sulfoxide), Life Technologies) in $0.1 \mathrm{M}$ phosphate buffer at $\mathrm{pH} 7.2$ was applied and incubated (2 hrs at RT). The solution was then removed and replaced with test compounds at concentrations from $1 \mathrm{mM}$ to 1 $\mathrm{nM}$ ) followed by incubation (2 hrs at RT). The treated wells were then subjected to CSLM (Leica TCS SP5 with 405nm excitation of PDMPO) with detection of fluorescence between $480 \mathrm{~nm}$ and $600 \mathrm{~nm}$ along the z-axis. Instrumental parameters were: the numerical aperture (0.5 NA), objectives (Leica HCX PL Fluotar, 20×), pinhole size ( $84 \mu \mathrm{m}$ ) and step size of $0.5 \mu \mathrm{m}$, PMT gain (1107 V), offset (2.7\%) and laser intensity (77\%). If not stated otherwise, a minimum of three intensity curves were averaged for each inhibitor molecule concentration and normalized to the maximum intensities. All reagents used, aniline hydrochloride, ammonium persulfate, glutaric dialdehyde ( $50 \% \mathrm{v} / \mathrm{v})$, amino acids (alanine, histidine and aspartic acid), lysozyme, tetramethyl 
orthosilicate (TMOS), polyamine (spermine), hydrochloric acid, sodium hydroxide, ibuprofen, monobasic potassium phosphate monohydrate and dibasic potassium phosphate were obtained from Sigma-Aldrich.

Zinc oxide: Single-crystalline ZnO (0001)-Zn and (10-10) substrates (Crystal GmBH, Berlin, Germany) were fixed in a 96 microwell plate after 30 min cleaning treatment with an UV Ozone Cleaner (ProCleaner Plus, BioForce Nanosciences). To determine the binding constant of the FluoZin-1 Dye (F24181, Thermo Fisher) to the ZnO surfaces, we measured the concentrationdependent adsorption behavior and the time-dependent increase of the fluorescence intensity upon interaction with the $\mathrm{ZnO}$ (0001)-Zn and (10-10) substrates. Binding studies were initiated by adding $200 \mu \mathrm{l}$ of $10 \mu \mathrm{M}$ FluoZin-1 dye in methanol and incubating (2 hr) on a shaker at $30 \mathrm{rpm}$ at room temperature. The dye-containing solution was removed and replaced with $200 \mu$ methanol (control) or with $200 \mu 1$ solutions ( $1 \mathrm{nM}$ to $1 \mathrm{mM}$ ) containing test compounds (the amino acids, $\mathrm{H}$, C, S, L, A and P, Sigma-Aldrich) and incubated (2 hr) using the same conditions as above. Note: all the $\mathrm{ZnO}$ interaction studies were performed in methanol to avoid the rapid dissolution of $\mathrm{ZnO}$ single crystalline substrates in aqueous media. ${ }^{54}$ The substrates were measured using CSLM (Leica TCS SP5) using $496 \mathrm{~nm}$ excitation of FluoZin-1) with detection of fluorescence between $498 \mathrm{~nm}$ and $601 \mathrm{~nm}$ along the $\mathrm{z}$-axis. Instrumental parameters were: the numerical aperture (0.5 NA), objectives (Leica HCX PL Fluotar, 10×), pinhole size (84 $\mu \mathrm{m}$ ) and step size of $1 \mu \mathrm{m}$, PMT gain (1107 V), offset (2.7\%) and laser intensity (67\%). If not stated otherwise, a minimum of three intensity curves were averaged for each inhibitor molecule concentration and normalized to the maximum intensities.

Data fitting: The assay results were fitted using a modification of the generalized logistic function using commercially available software (Origin; OriginLab 2015) using a Levenberg-Marquardt 
algorithm with a tolerance of $10^{-9}$ and maximum number of 400 iterations. The resulting curve is plotted along with the 95\% confidence intervals and the 95\% prediction intervals.

\section{Theory}

This study adapts two similar fluorescence-based indicator displacement assays. ${ }^{18,19}$ One is the optically sectioned planar format assay (OSPFA), which has been described previously for antibodies and receptors (Figure 1a and e). The other involves selection of the indicator such that fluorescence is lost upon displacement (Figure $1 \mathrm{~b}$ and f). Choosing an indicator with high affinity and specificity towards the surface of interest under the desired solvent conditions is key for this assay. For instance, PDMPO is known to be relatively insensitive to the presence of different ions and ionic strength ${ }^{55}$ and FluoZin-1 was developed for a cellular environment. These surface-bound assays are based on a two-step process: (i) the indicator is brought into contact with the surface where it binds reversibly (Figure $1 \mathrm{a}$ and $\mathrm{b}$ ) and (ii) a competitive molecule is added at increasing concentration (Figure $1 \mathrm{e}$ and $\mathrm{f}$ ) to test its ability to displace the indicator. If the test molecule causes the indicator to move from the surface into solution this response can be detected. ${ }^{18,19}$ For studies involving PDMPO as the indicator, PDMPO is found both bound to the surface and in bulk solution and the contributions of each have to be isolated from the observed spectral response. The approach to separate out the value of interest is described in the supplementary information and Supplementary Figure 1. The surface-bound contribution is the quantity of interest for subsequent $\mathrm{EC}_{50}$ and $\mathrm{IC}_{50}$ determinations. 
a)

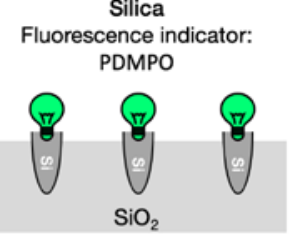

e) $(\overbrace{\mathrm{SiO}_{2}}^{\text {(Bio-) Molecule }}$ b)
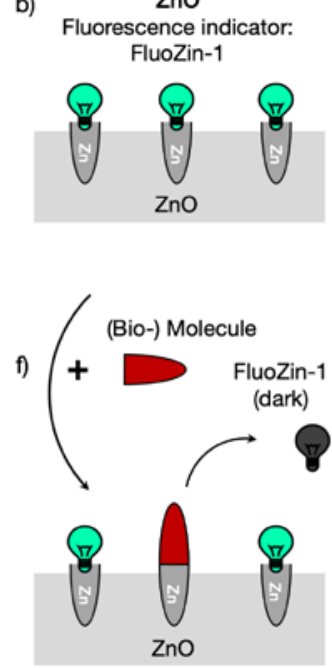

c)

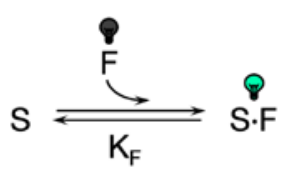

d)

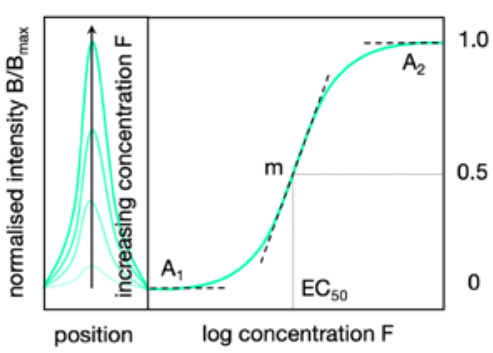

g)

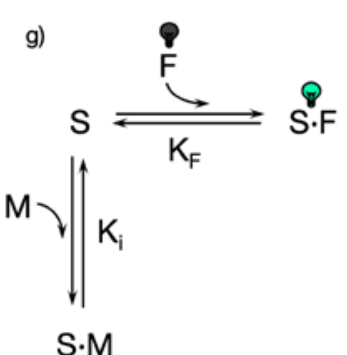

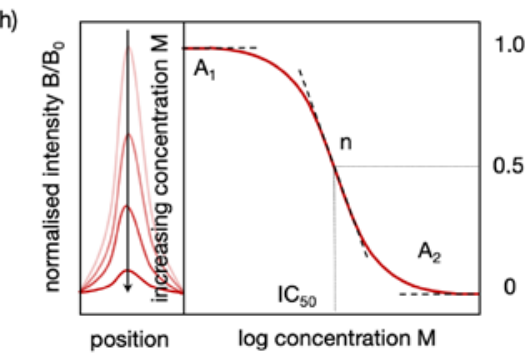

Figure 1: Binding assays of the selected indicators (upper panel) and comparison of the optically sectioned planar format indicator displacement assay for silica and $\mathrm{ZnO}$ (lower panel). a) and e) Schematic representation of the interaction at the silica interface ('reduced' indicates a reduction in signal intensity) and b) and f) at the ZnO interface. c) and g) Model reactions and their equilibrium constants during the binding assay and the displacement assay, respectively. S corresponds to the number of binding sites on the surface, $\mathrm{F}$ denotes the fluorescent dye and $\mathrm{M}$ the molecule of interest d) Concentration-dependent increase of the normalized fluorescent thin layer intensity $\mathrm{B} / \mathrm{B}_{\max }$, fitted by a modification of the generalized logistic function, leading to four fitting parameters including two asymptotes $\left(A_{1}, A_{2}\right)$, the slope $\mathrm{m}$ as well as the concentration producing $50 \%$ of the maximal response $\left(\mathrm{EC}_{50}\right)$. h) Concentration-dependent decrease of the normalized fluorescent thin layer intensity $\mathrm{B} / \mathrm{B}_{0}$ fitted by a modification of the generalized logistic function, leading to four fitting parameters including the two asymptotes $\left(A_{1}, A_{2}\right)$, the slope $\mathrm{n}$ as well as the concentration producing a $50 \%$ reduction in the response $\left(\mathrm{IC}_{50}\right)$.

The use of dyes like FluoZin-1 ${ }^{56,57}$ simplifies the treatment as FluoZin-1 becomes brightly fluorescent when it binds to $\mathrm{Zn}^{2+}$ in solution or to the coordinated zinc species in the surface of the $\mathrm{ZnO}$ single crystals used in this study; however, it is non-fluorescent when displaced from zinc species by a test compound. ${ }^{56}$ Thus one can simply use the optical response along the $z$-axis perpendicular to the surface to obtain intensity values which are used directly in equations 1 and 
2 for the $\mathrm{EC}_{50}$ and $\mathrm{IC}_{50}$ determinations. The approach is adaptable to non-planar sample shapes; however, the planar shape is useful as it allows $\mathrm{ZnO}$ single crystals with specific orientation, in this case (0001)-Zn and (10-10) surfaces, to be assessed.

Binding affinities of the indicator. For the optically sectioned planar format indicator displacement assay (O-IDA), the interaction of the indicator, in our case the fluorescent dyes PDMPO and FluoZin-1, with the substrate, silica and ZnO, respectively, must be calculated based on a binding assay before we can elucidate the interaction of the inhibitor molecules. This binding assay describes the formation of the fluorescent dye layer (thin layer) on the substrate. Upon maximum coverage of the substrate with the indicator a saturation of the maximum fluorescence intensity is observed, and addition of further indicator molecules does not contribute to the measured thin layer intensity meaning it does not alter the binding equilibrium.

Calculation of $\mathbf{E C}_{50}$. Here we consider $\mathrm{EC}_{50}$ as the concentration required to reach half saturation of the surface with indicator. The values are obtained by fitting the indicator/ dye concentration and the associated intensities to a logistic dose-response equation:

$$
\frac{B}{B_{\max }}=A_{1}+\frac{A_{2}-A_{1}}{1+10^{\left(x-\log x_{0}\right) m} .}
$$

Plotting the fluorescence intensity $\mathrm{B}$ against the logarithm of the concentration $\mathrm{x}$ results in a sigmoidal shape for any value of the slope of the dose response curve $m . A_{1}$ and $A_{2}$ describe the asymptotes, $x_{0}$ corresponds to the sigmoid midpoint, which gives the EC $\mathrm{F}_{50}$ values as $10^{x_{0}}$.

If fluorescence intensities are normalized to $B_{\max }, A_{2}=1$ and in the case where there is no residual background, $A_{1}$ simplifies to 0 . The surface specific binding constant $K_{F}$ of the fluorescence dye depends on the slope, $m$, of the curves via $K_{F}=E C_{50}^{m}$. When $m=1, \mathrm{EC}_{50}$ would be equivalent to the dissociation constant of the fluorescence dye from the surface $\left(K_{F}\right)$. 
Indicator displacement assay: To perform the indicator displacement assay, we firstly remove the supernatant including the non-bonded indicator molecules and replace it with a solvent to access the fluorescence intensity after an incubation period. This fluorescence signal is the initial state for the following displacement assay and provides the reference intensity $\left(\mathrm{B}_{0}\right)$. For the displacement assay, the compound of interest is added in solution at different concentrations and the fluorescence signal is measured after an equilibration period and compared to the initial state. After full replacement of the dye, additional binding would not be detected for instance for the formation of multilayers.

Calculation of $\mathbf{I C}_{\mathbf{5 0}}$. During the indicator displacement assay, we access the so-called $\mathrm{IC}_{50}$ value. Here, $\mathrm{IC}_{50}$ is defined as the concentration required to displace $50 \%$ of the bound indicator molecules in the thin layer from the initial state. $\mathrm{IC}_{50}$ is determined from the concentration dependent fluorescence of the indicator on the surface (c.f. Figure 1) and describes the potency of an assayed test compound. Modifying the generalized logistic function in equation 1 to account for a displacement by changing the sign of the exponent yields:

$$
\frac{B}{B_{0}}=A_{2}+\frac{A_{1}-A_{2}}{1+10^{-\left(x-\log x_{0}\right) n}}
$$

where the parameters are the same as in equation 1 . Here the fits were simplified by applying a one-site competition curve, which is a dose response curve with a Hill slope $n$ equal to 1 . The $\mathrm{IC}_{50}$ is then obtained from $10^{x_{0}}$. Here, $A_{1}$ and $A_{2}$ can be used to account for residual fluorescence not treated already and to account for irreversible binding of the indicator dye.

Calculation of $\mathbf{K}_{\mathbf{i}}$. A quantity analogous to the inhibitory binding constant, $K_{\mathrm{i}},{ }^{58}$ can be defined for these assays. A range of approaches have been considered, ${ }^{58-62}$ however, the approach from Cheng, ${ }^{61,62}$ who highlighted the importance of the slope function, appears to be the most suitable for our treatment since it is obtained directly from the law of mass. ${ }^{61,62}$ This approach includes the 
slopes $n$ and $m$ for the antagonist (substrate of interest) and agonist (fluorescent dye), respectively, and gives:

$$
K_{i}=\frac{I C_{50}{ }^{n}}{1+\left(\frac{[F]}{E C_{50}}\right)^{m}}=\frac{I C_{50}{ }^{n}}{1+\frac{[F]^{m}}{K_{F}}} .
$$

As noted before, in the current study we assume that $\mathrm{n}=1$.

Calculation of the free energy of fluorescent dye binding $\Delta G_{F}$ and adsorption $\Delta G_{a d s}$. The availability of an equilibrium dissociation constant for the binding of the fluorescent dye, $K_{F}$, allows for the calculation of the binding affinity following $\Delta G_{F}=-R T \ln \left(\bar{v}_{S} \cdot K_{F}\right)$ with $\bar{v}_{s}$ being the partial molar volume of the solvent used, ${ }^{63}$ in our cases $\bar{v}_{s}=0.018 \mathrm{l} \mathrm{mol}^{-1}$ for the aqueous environment and $\bar{v}_{s}=0.04046 \mathrm{l} \mathrm{mol}^{-1}$ for the methanol environment. The free energy of adsorption is calculated similarly, $\Delta G_{a d s}=-R T \ln \left(\bar{v}_{s} \cdot K_{i}\right)$, with $K_{i}$ being the inhibitory binding constant.

\section{Results and discussion}

The silica system is studied in aqueous media in accordance with previous binding studies ${ }^{1}$ and conditions used for both studies of biomineralization and drug delivery. The $\mathrm{ZnO}$ system is studied in methanol to prevent dissolution ${ }^{54}$ and to compare to conditions used in some bio-mineralization strategies ${ }^{64,65}$ The usage of different solvent systems showcases the versatility of the approach. 


\section{Silica/PDMPO interaction in an optically sectioned planar format indicator displacement}

assay

Measurement of the binding of the PDMPO fluorescent dye in phosphate buffer to silica (Supplementary Figure 2) yield an $\mathrm{EC}_{50}$ value of $1.75 \pm 0.27 \mu \mathrm{M}, K_{F}=0.01 \pm 0.02 \mu \mathrm{M}$ and a binding energy $\Delta G_{F}=-13.28 \pm 0.12 \mathrm{kcal} \mathrm{mol}^{-1}$.

The assay system was then challenged with the amino acids alanine, histidine and aspartic acid, the polyamine spermine, and ibuprofen. These specific amino acids were chosen as they are commonly found in peptides that bind to silica. ${ }^{66} \mathrm{~A}$ polyamine was selected as it has been found to be part of the post-translational modifications of proteins isolated from silicifying organisms such as some diatoms. ${ }^{67,68}$ It has also been shown to moderate silica formation in vitro. ${ }^{69,70}$ Ibuprofen was selected as an example of a commonly used hydrophobic drug which could serve as a test case for silica-based drug delivery systems that can control the release of drugs and deliver them to specific targets.

The dose response behaviour of the selected biomolecules are presented in Figure 2 (exemplarily for histidine) and in Supplementary Figure 3 (for all other systems). The calculated $\mathrm{IC}_{50}, K_{i}$ and $\Delta G_{a d s}$ values are presented in Table 1 . Of the compounds tested, ibuprofen had the strongest affinity for the silica surface and spermine the weakest. The binding energies covered the range from -12.82 to $-9.39 \mathrm{kcal} \mathrm{mol}^{-1}$ with the affinity of amino acids towards hydroxylated silica being: histidine>aspartic acid $>$ alanine, this trend aligns with a computational study that studied the binding affinities of several amino acids to an amorphous hydroxylated silica surface with relatively different hydrophilicity performed under gas conditions. ${ }^{68}$ 
a) Histidine on Silica

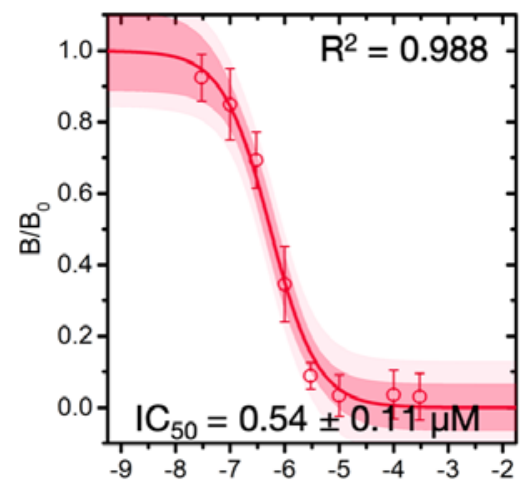

$\log (\mathrm{M})$ b) Histidine on $\mathrm{ZnO}$ (0001)- $\mathrm{Zn}$

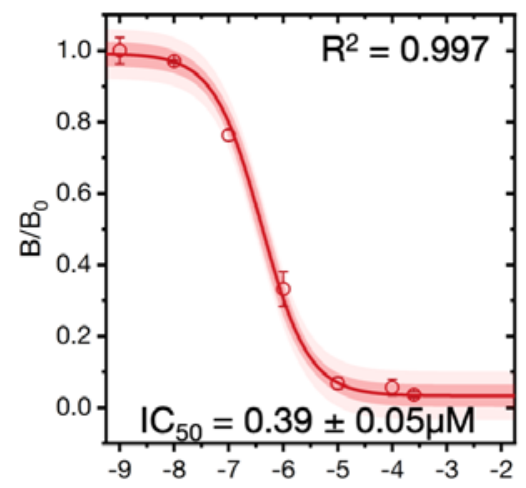

$\log (\mathrm{M})$
Histidine on $\mathrm{ZnO}(10-10)$

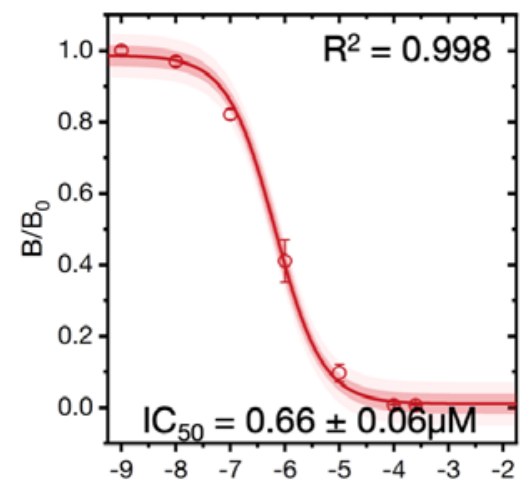

$\log (\mathrm{M})$

Figure 2: Exemplar dose response curves from histidine a) with silica, b) with the (0001)-Zn, and c) with (10-10) ZnO single crystal surfaces.

Histidine's highest binding affinity to silica most likely includes interactions of the imidazole side chain interacting with silanol/siloxide groups on the silica surface as has been reported for peptides containing histidine. ${ }^{71,72}$ We propose that protonated $\mathrm{N}$ atoms of the imidazole ring may form direct $\mathrm{H}$ bonds with the silanol terminal group of the silica surface, in particular with deprotonated silanols that would be present at the $\mathrm{pH}$ of the experiment. Another contribution to binding will arise when the imidazole ring inserts flat into locally hydrophobic pockets while still forming hydrogen bonds with terminal $\mathrm{OH}$ groups. Both behaviours have been observed for peptide silica binding. ${ }^{71,72}$

For the other two investigated amino acids, we obtain similar values for their binding affinity towards silica. With respect to the very different side functionalities, we would expect a common binding configuration via the amine group, which is known from molecular dynamics simulations on silica. ${ }^{71,73}$

We note that computational studies of isolated amino acid binding to silica have only been performed on different surfaces of fully hydroxylated quartz in vacuum or for amino acid 
analogues on fully hydroxylated quartz in an aqueous environment; thus there is no directly comparable computational data available. Of the data that is available, computed adsorption enthalpies $\left(\Delta E_{a d s}\right)$ for alanine and aspartic acid on the (10-10) surface of quartz range from ca. -17 to $-20 \mathrm{kcal} \mathrm{mol}^{-1} \cdot{ }^{74,75}$ For studies of 'models of amino acids' (e.g. methane for alanine; methanol for serine; butylamine for lysine and acetic acid for glutamic acid) the values are all around the -1 to $-2 \mathrm{kcal} \mathrm{mol}^{-1}$ range. ${ }^{76}$ For the investigated polyamine, ${ }^{77}$ the interaction with silica will most probably be mediated by the amine groups, leading to a comparable binding affinity. Similarly, there are no computational data available for comparison.

Table 1: Binding affinities of selected biomolecules to silica surfaces. The standard error of the fitting for the IC50 values were propagated during the calculations.

\begin{tabular}{|c|c|c|c|c|}
\hline \multicolumn{2}{|c|}{ Name } & $\begin{array}{l}\mathrm{IC}_{50} \\
(\mu \mathrm{M})\end{array}$ & $\begin{array}{c}\mathrm{K}_{\mathrm{i}} \\
(\mu \mathrm{M})\end{array}$ & $\begin{array}{c}\Delta \mathbf{G}_{\text {ads }} \\
\left(\mathrm{kcal} \mathrm{mol}^{-1}\right)\end{array}$ \\
\hline \multirow{3}{*}{$\begin{array}{l}\text { Amino } \\
\text { acids }\end{array}$} & Alanine & $6.17 \pm 1.77$ & $4.23 \pm 1.30$ & $-9.71 \pm 0.18$ \\
\hline & Histidine & $0.54 \pm 0.11$ & $0.37 \pm 0.08$ & $-11.15 \pm 0.12$ \\
\hline & $\begin{array}{l}\text { Aspartic } \\
\text { acid }\end{array}$ & $3.43 \pm 0.71$ & $2.35 \pm 0.52$ & $-10.06 \pm 0.13$ \\
\hline Polyamine & Spermine & $10.7 \pm 2.0$ & $7.33 \pm 1.45$ & $-9.39 \pm 0.12$ \\
\hline Drugs & Ibuprofen & $0.032 \pm 0.003$ & $0.022 \pm 0.003$ & $-12.82 \pm 0.08$ \\
\hline
\end{tabular}


In our experimental study, Ibuprofen was found to exhibit the highest binding affinity for silica amongst all studied binding partners, with $\Delta G_{a d s}=-12.82 \pm 0.08 \mathrm{kcal} \mathrm{mol}^{-1}$. In recent density functional theory (DFT) simulations, ${ }^{32,78}$ interactions of ibuprofen with an hydroxylated silica surface were indeed shown to be exothermic and exergonic. Weak hydrogen-bonding between the carboxylic functionality of ibuprofen and $\mathrm{OH}$ surface groups of silica surface and dispersive attractions between the non-polar portion of ibuprofen and the surface were both found to be important, ${ }^{32}$ particularly in the presence of added water molecules. ${ }^{78}$

\section{ZnO/FluoZin indicator displacement assay}

The facet-specific binding of FluoZin-1 to $\mathrm{ZnO}$ in methanol (Supplementary Figure 4 and Supplementary Table 1) indicates that binding is stronger to the polar terminated $\mathrm{ZnO}(0001)-\mathrm{Zn}$ $\left(\left(\Delta G_{F}=-16.32 \pm 0.03 \mathrm{kcal} \mathrm{mol}^{-1}\right)\right.$ than to the mixed terminated $(10-10)$ surface $\left(\Delta G_{F}=-14.58 \pm\right.$ $0.05 \mathrm{kcal} \mathrm{mol}^{-1}$ ). For the investigation of the binding affinities of amino acids we fitted the dose response behaviour with equation 2, similar to the Silica/PDMPO system (Figure 2, Supplementary Figure 5 and Supplementary Figure 6). The results are summarized in Table 2. The binding affinities of the amino acids to the (0001)-Zn surface can be ranked in the following order: histidine $\gtrsim$ proline $>$ serine $\gtrsim$ cysteine $>$ leucine $\gtrsim$ alanine. For the amino acid/(10-10) ZnO interface, the ranking of the amino acids leads to a slightly different order: proline $\gtrsim$ histidine $>$ serine $\gtrsim$ cysteine $>$ alanine $\gtrsim$ leucine.

The interaction of histidine and proline is characterized by very similar values of the binding free energy within our experimental setup (c.f. Table 2). While the interaction of $\mathrm{Zn}^{2+}$ ions in solution with histidine ${ }^{79-81}$ and within zinc finger proteins ${ }^{46,48,82-84}$ has been extensively 
investigated, little is known about the interaction with proline. Biomineralization studies with amino acids support the strong influence of cysteine and histidine residues on the morphology of $\mathrm{ZnO}$ particles, while the influence of proline was less pronounced. ${ }^{85,86}$

We hypothesize that the interaction of histidine and proline may take place through a qualitatively similar binding mode, namely via the nitrogen of the imidazole ring and the pyrrolidine group, respectively. However, the precise interaction patterns will very likely be determined by the local arrangement of the surface's terminal groups together with the exact protonation states of both the amino acids and the surface. Such protonation states in a pure methanol environment are not unambiguously accessible at this point and should be investigated carefully in future combined experimental/theoretical studies.

Table 2: Binding affinities of selected amino acids to the polar (0001)-Zn and the mixed terminated (10-10) surfaces of $\mathrm{ZnO}$. The standard error of the fitting for the IC50 values were propagated during the calculations.

\begin{tabular}{|c|c|c|c|c|}
\hline \multicolumn{2}{|c|}{ Name } & $\begin{array}{l}\mathrm{IC}_{50} \\
(\mu \mathrm{M})\end{array}$ & $\begin{array}{c}\mathrm{K}_{\mathrm{i}} \\
(\mu \mathrm{M})\end{array}$ & $\begin{array}{c}\Delta G_{a d s} \\
\left(\mathrm{kcal} \mathrm{mol}^{-1}\right)\end{array}$ \\
\hline \multirow{2}{*}{ Alanine } & (0001)-Zn & $461 \pm 23$ & $6.94 \pm 2.19$ & $-8.94 \pm 0.19$ \\
\hline & $(10-10)$ & $153 \pm 28$ & $3.77 \pm 0.98$ & $-9.30 \pm 0.15$ \\
\hline \multirow{2}{*}{ Cysteine } & (0001)-Zn & $53.2 \pm 4.5$ & $0.80 \pm 0.26$ & $-10.22 \pm 0.19$ \\
\hline & $(10-10)$ & $62.6 \pm 6.4$ & $1.55 \pm 0.33$ & $-9.83 \pm 0.13$ \\
\hline
\end{tabular}




\begin{tabular}{|c|c|c|c|c|}
\hline \multirow{2}{*}{ Histidine } & (0001)-Zn & $0.39 \pm 0.05$ & $0.006 \pm 0.002$ & $-13.12 \pm 0.20$ \\
\hline & $(10-10)$ & $0.66 \pm 0.06$ & $0.016 \pm 0.003$ & $-12.53 \pm 0.13$ \\
\hline \multirow{2}{*}{ Proline } & (0001)-Zn & $1.52 \pm 0.07$ & $0.023 \pm 0.007$ & $-12.33 \pm 0.19$ \\
\hline & $(10-10)$ & $0.55 \pm 0.06$ & $0.014 \pm 0.003$ & $-12.63 \pm 0.13$ \\
\hline \multirow{2}{*}{ Serine } & (0001)-Zn & $48.6 \pm 4.7$ & $0.73 \pm 0.24$ & $-10.27 \pm 0.19$ \\
\hline & $(10-10)$ & $101 \pm 5$ & $2.49 \pm 0.48$ & $-9.55 \pm 0.12$ \\
\hline \multirow{2}{*}{ Leucine } & (0001)-Zn & $375 \pm 28$ & $5.64 \pm 1.81$ & $-9.06 \pm 0.19$ \\
\hline & $(10-10)$ & $231 \pm 17$ & $5.71 \pm 1.15$ & $-9.05 \pm 0.12$ \\
\hline
\end{tabular}

In agreement with previous research, we obtain similar binding affinities for the amino acids cysteine and serine. ${ }^{87}$ Though a proton-transfer to the surface was proposed for the adsorption of cysteine on the (10-10) surface, ${ }^{87}$ in agreement with the proposed interactions within zinc finger proteins, the interaction of cysteine and serine on this surface was attributed to the direct or indirect formation of hydrogen bonds including the carboxylate and side chain groups. ${ }^{87}$ Presumably, the interaction of the aliphatic amino acids alanine ${ }^{88}$ and leucine with the $\mathrm{ZnO}$ surfaces is via their cterminal carboxylate groups alone, leading to the lowest binding affinities obtained in our experimental study.

\section{Conclusions}


This study reports the first measurements of small molecule interactions with silica and zinc oxide surfaces using optically sectioned indicator displacement assays (O-IDAs). As demonstrated using the $\mathrm{ZnO}$ system using methanol as the solvent, these O-IDAs are not restricted to aqueous systems or materials that are insoluble in an aqueous environment. They can be performed in any solvent and with any material provided a suitable dye is available and the system overall does not produce interfering fluorescence. Additionally, investigation of interaction kinetics may be possible provided that the interactions investigated happen on a timescale slower than the time of image acquisition in the confocal microscope.

The transfer of traditional indicator displacement assays to study the abiotic/biotic interface also requires us to keep in mind the underlying receptor occupancy theory with its assumptions. ${ }^{89-92}$ For a direct comparison, we do not know the number of accessible binding sites on our surface of interest, for instance due to surface protonation and system dynamics. In the framework of abiotic/biotic interactions, the maximum number of binding sites corresponds to the number that can be occupied by a known concentration of fluorescent dye. However, it is important to note that steric hindrance might prevent full occupancy. Further, during the displacement assay also nonindicator-occupied binding sites might be accessible and additionally not all analytes interact at the same binding site as the fluorescent dye due to the different chemistry involved. For instance, the dye FluoZin-1 binds to the zinc surface species, while some of the investigated amino acids will probably interact with the molecular adsorbed and/or dissociated water molecules present on the surface; the dye would in this case be displaced due to 'steric' considerations.

The O-IDA approach introduced here could be used similarly to the fluorescence-based indicator displacement assays in pharmacological screening since the method is convenient, robust, and scalable to high-throughput formats. In this form, O-IDAs could be of interest for assessing the 
binding of medicinally relevant peptides or substrates for catalytic applications. This technique has advantages over quartz crystal microbalance (QCM) and surface plasmon resonance approaches by avoiding the issues associated with isolating the behaviour of molecules versus solvent/ions (QCM) and the need for a conductive surface (SPR). The only limitation of the OIDA technique is the requirement for a fluorescent dye that has a specific interaction towards the abiotic surface of interest. A major advantage in comparison to the alternative approaches is the direct visualization of the adsorption events, which allows for quality control judgements regarding the homogeneity and spatial distributions of the response.

\section{ASSOCIATED CONTENT}

\section{Supporting Information.}

The following files are available free of charge:

Synthesis of the functionalised silica surfaces; Overview of the Silica/PDMPO interaction in a planar format indicator assay; Characterization of the fluorescent dye PDMPO; O-IDA results for amino acids, ibuprofen and spermine on Silica using PDMPO; Characterization of the fluorescent dye FluoZin-1; Binding affinity of FluoZin-1 towards ZnO (0001)-Zn and (10-10); O-IDA results for amino acids on $\mathrm{ZnO}$ (0001)-Zn using FluoZin-1; O-IDA results for amino acids on $\mathrm{ZnO}(10-10)$ using FluoZin-1.

\section{AUTHOR INFORMATION}

\section{Corresponding Author}

* carole.perry@ntu.ac.uk, 


\section{Author Contributions}

The manuscript was written through contributions of all authors. All authors have given approval to the final version of the manuscript. ¥These authors contributed equally.

\section{Funding Sources}

CCP acknowledges Airforce office of scientific research FA9550-13-1-0040 and FA9550-16-1-0213. LCC acknowledges funding from the Deutsche Forschungsgemeinschaft under Grants No. CO 1043/17- 1 and CO 1043/11-1.

\section{ACKNOWLEDGMENT}

The authors thank Dr. Massimo Delle Piane for fruitful discussions and Mr Daniel Oliver for dose response measurements for $\mathrm{ZnO}$.

\section{ABBREVIATIONS}

CSLM: Confocal scanning laser microscopy; IDA: indicator displacement assay; OSPFA: optically sectioned planar format assay; SPR: surface plasmon resonance; QCM: quartz crystal microbalance 


\section{REFERENCES}

(1) Sola-Rabada, A.; Michaelis, M.; Oliver, D. J.; Roe, M. J.; Colombi Ciacchi, L.; Heinz, H.; Perry, C. C. Interactions at the Silica-Peptide Interface: Influence of the Extent of Functionalization on the Conformational Ensemble. Langmuir 2018, 34 (28), 8255-8263. https://doi.org/10.1021/acs.langmuir.8b00874.

(2) Patwardhan, S. V.; Patwardhan, G.; Perry, C. C. Interactions of Biomolecules with Inorganic Materials: Principles, Applications and Future Prospects. J. Mater. Chem. 2007, 17 (28), 2875-2884. https://doi.org/10.1039/b704075j.

(3) Puddu, V.; Perry, C. C. Peptide Adsorption on Silica Nanoparticles: Evidence of Hydrophobic Interactions. ACS Nano 2012, $6 \quad$ (7), 6356-6363. https://doi.org/10.1021/nn301866q.

(4) Hildebrand, N.; Michaelis, M.; Wurzler, N.; Li, Z.; Hirst, J. D.; Micsonai, A.; Kardos, J.; Gil-Ley, A.; Bussi, G.; Köppen, S.; et al. Atomistic Details of Chymotrypsin Conformational Changes upon Adsorption on Silica. ACS Biomater. Sci. Eng. 2018, 4 (12), 4036-4050. https://doi.org/10.1021/acsbiomaterials.8b00819.

(5) Anselme, K.; Davidson, P.; Popa, A. M.; Giazzon, M.; Liley, M.; Ploux, L. The Interaction of Cells and Bacteria with Surfaces Structured at the Nanometre Scale. Acta Biomater. 2010, 6 (10), 3824-3846. https://doi.org/10.1016/j.actbio.2010.04.001.

(6) Nel, A. E.; Mädler, L.; Velegol, D.; Xia, T.; Hoek, E. M. V; Somasundaran, P.; Klaessig, F.; Castranova, V.; Thompson, M. Understanding Biophysicochemical Interactions at the Nano--Bio Interface. Nat. Mater. 2009, 8 (7), 543-557. https://doi.org/10.1038/nmat2442. 
(7) Roach, P.; Farrar, D.; Perry, C. C. Interpretation of Protein Adsorption: Surface-Induced Conformational Changes. J. Am. Chem. Soc. 2005, 127 (22), 8168-8173. https://doi.org/10.1021/ja042898o.

(8) Roach, P.; Eglin, D.; Rohde, K.; Perry, C. C. Modern Biomaterials: A Review—Bulk Properties and Implications of Surface Modifications. J. Mater. Sci. Mater. Med. 2007, 18 (7), 1263-1277. https://doi.org/10.1007/s10856-006-0064-3.

(9) Hench, L. L. Biomaterials: A Forecast for the Future. Biomaterials 1998, 19 (16), 14191423. https://doi.org/10.1016/S0142-9612(98)00133-1.

(10) Williams, D. F. On the Nature of Biomaterials. Biomaterials 2009, 30 (30), 5897-5909. https://doi.org/10.1016/j.biomaterials.2009.07.027.

(11) Li, Q.; Michaelis, M.; Wei, G.; Colombi Ciacchi, L. A Novel Aptasensor Based on SingleMolecule Force Spectroscopy for Highly Sensitive Detection of Mercury Ions. Analyst 2015, 140 (15), 5243-5250. https://doi.org/10.1039/C5AN00708A.

(12) Li, Q.; Zhang, T.; Pan, Y.; Ciacchi, L. C.; Xu, B.; Wei, G. AFM-Based Force Spectroscopy for Bioimaging and Biosensing. RSC Adv. 2016, 6 (16), 12893-12912. https://doi.org/10.1039/C5RA22841G.

(13) O’Sullivan, C. K.; Guilbault, G. G. Commercial Quartz Crystal Microbalances - Theory and Applications. Biosens. Bioelectron. 1999, 14 (8-9), 663-670. https://doi.org/10.1016/S0956-5663(99)00040-8.

(14) Homola, J.; Yee, S. S.; Gauglitz, G. Surface Plasmon Resonance Sensors: Review. Sensors 
Actuators B Chem. 1999, 54 (1-2), 3-15. https://doi.org/10.1016/S0925-4005(98)00321-9.

(15) Dudko, O. K.; Filippov, A. E.; Klafter, J.; Urbakh, M. Beyond the Conventional Description of Dynamic Force Spectroscopy of Adhesion Bonds. Proc. Natl. Acad. Sci. 2003, 100 (20), 11378-11381. https://doi.org/10.1073/pnas.1534554100.

(16) Freire, E.; Mayorga, O. L.; Straume, M. Isothermal Titration Calorimetry. Anal. Chem. 1990, 62 (18), 950A-959A. https://doi.org/10.1021/ac00217a002.

(17) Hennig, A.; Bakirci, H.; Nau, W. M. Label-Free Continuous Enzyme Assays with Macrocycle-Fluorescent Dye Complexes. Nat. Methods 2007, 4 (8), 629-632. https://doi.org/10.1038/nmeth1064.

(18) Nguyen, B. T.; Wiskur, S. L.; Anslyn, E. V. Using Indicator-Displacement Assays in Test Strips and to Follow Reaction Kinetics. Org. Lett. 2004, 6 (15), 2499-2501.

(19) Nguyen, B. T.; Anslyn, E. V. Indicator-Displacement Assays. Coord. Chem. Rev. 2006, 250 (23-24), 3118-3127. https://doi.org/10.1016/j.ccr.2006.04.009.

(20) Ghafari, H.; Parambath, M.; Hanley, Q. S. Macromolecular Binding and Kinetic Analysis with Optically Sectioned Planar Format Assays. Analyst 2012, 137 (20), 4809-4814. https://doi.org/10.1039/c2an35134j.

(21) Barbé, C.; Bartlett, J.; Kong, L.; Finnie, K.; Lin, H. Q.; Larkin, M.; Calleja, S.; Bush, A.; Calleja, G. Silica Particles: A Novel Drug-Delivery System. Adv. Mater. 16 (21), 19591966. https://doi.org/10.1002/adma.200400771.

(22) Delle Piane, M.; Corno, M.; Ugliengo, P. Ab Initio Modeling of Hydrogen Bond Interaction 
at Silica Surfaces With Focus on Silica/Drugs Systems. In Modelling and Simulation in the Science of Micro- and Meso-Porous Materials; Catlow, C. R. A., Speybroeck, V. Van, van Santen, R. A., Eds.; Elsevier, 2018; pp 297-328. https://doi.org/10.1016/B978-0-12805057-6.00009-0.

(23) Sola-Rabada, A.; Sahare, P.; Hickman, G. J.; Vasquez, M.; Canham, L. T.; Perry, C. C.; Agarwal, V. Biogenic Porous Silica and Silicon Sourced from Mexican Giant Horsetail (Equisetum Myriochaetum) and Their Application as Supports for Enzyme Immobilization. Colloids Surfaces B Biointerfaces 2018, 166, 195-202. https://doi.org/10.1016/j.colsurfb.2018.02.047.

(24) Townson, J. L.; Lin, Y.-S.; Chou, S. S.; Awad, Y. H.; Coker, E. N.; Brinker, C. J.; Kaehr, B. Synthetic Fossilization of Soft Biological Tissues and Their Shape-Preserving Transformation into Silica or Electron-Conductive Replicas. Nat. Commun. 2014, 5, 5665. https://doi.org/10.1038/ncomms6665.

(25) Kaehr, B.; Townson, J. L.; Kalinich, R. M.; Awad, Y. H.; Swartzentruber, B. S.; Dunphy, D. R.; Brinker, C. J. Cellular Complexity Captured in Durable Silica Biocomposites. Proc. Natl. Acad. Sci. 2012, 109 (43), 17336-17341. https://doi.org/10.1073/pnas.1205816109.

(26) Kröger, N.; Deutzmann, R.; Sumper, M. Polycationic Peptides from Diatom Biosilica That Direct Silica Nanosphere Formation. Science (80-. ). 1999, 286 (5442), 1129-1132. https://doi.org/10.1126/science.286.5442.1129.

(27) Kroger, N.; Deutzmann, R.; Bergsdorf, C.; Sumper, M. Species-Specific Polyamines from Diatoms Control Silica Morphology. Proc. Natl. Acad. Sci. 2000, 97 (26), 14133-14138. 
https://doi.org/10.1073/pnas.260496497.

(28) Hildebrand, M. Diatoms, Biomineralization Processes, and Genomics. Chem. Rev. 2008, 108 (11), 4855-4874. https://doi.org/10.1021/cr078253z.

(29) Pegg, A. E. Functions of Polyamines in Mammals. J. Biol. Chem. 2016, 291 (29), 1490414912. https://doi.org/10.1074/jbc.R116.731661.

(30) Salonen, J.; Laitinen, L.; Kaukonen, A. M.; Tuura, J.; Björkqvist, M.; Heikkilä, T.; VähäHeikkilä, K.; Hirvonen, J.; Lehto, V.-P. Mesoporous Silicon Microparticles for Oral Drug Delivery: Loading and Release of Five Model Drugs. J. Control. Release 2005, 108 (2-3), 362-374. https://doi.org/10.1016/j.jconrel.2005.08.017.

(31) Andersson, J.; Rosenholm, J.; Areva, S.; Lindén, M. Influences of Material Characteristics on Ibuprofen Drug Loading and Release Profiles from Ordered Micro- and Mesoporous Silica Matrices. Chem. Mater. 2004, 16 (21), 4160-4167. https://doi.org/10.1021/cm0401490.

(32) Delle Piane, M.; Corno, M.; Pedone, A.; Dovesi, R.; Ugliengo, P. Large-Scale B3LYP Simulations of Ibuprofen Adsorbed in MCM-41 Mesoporous Silica as Drug Delivery $\begin{array}{llllllll}\text { System. J. } & \text { Phys. } & \text { Chem. } & \text { C 2014, } & 118 & \text { (46), 26737-26749. }\end{array}$ https://doi.org/10.1021/jp507364h.

(33) Bowker, M. The Mechanism of Methanol Synthesis on Copper/Zinc Oxide/Alumina Catalysts. J. Catal. 1988, 109 (2), 263-273. https://doi.org/10.1016/0021-9517(88)902096. 
(34) Özgür, Ü.; Alivov, Y. I.; Liu, C.; Teke, A.; Reshchikov, M. A.; Doğan, S.; Avrutin, V.; Cho, S.-J.; Morkoç, H. A Comprehensive Review of ZnO Materials and Devices. J. Appl. Phys. 2005, 98 (4), 041301. https://doi.org/10.1063/1.1992666.

(35) Wang, Z. L. Zinc Oxide Nanostructures: Growth, Properties and Applications. J. Phys. Condens. $\quad$ Matter 2004, $16 \quad$ (25), R829-R858. https://doi.org/10.1088/09538984/16/25/R01.

(36) Zhang, Y.; Nayak, T.; Hong, H.; Cai, W. Biomedical Applications of Zinc Oxide Nanomaterials. Curr. Mol. Med. 2013, 13 (10), 1633-1645. https://doi.org/10.2174/1566524013666131111130058.

(37) Capper, P.; Kasap, S.; Willoughby, A. Zinc Oxide Materials for Electronic and Optoelectronic Device Applications; John Wiley \& Sons, 2011.

(38) Umetsu, M.; Mizuta, M.; Tsumoto, K.; Ohara, S.; Takami, S.; Watanabe, H.; Kumagai, I.; Adschiri, T. Bioassisted Room-Temperature Immobilization and Mineralization of Zinc Oxide-The Structural Ordering of ZnO Nanoparticles into a Flower-Type Morphology. Adv. Mater. 2005, 17 (21), 2571-2575. https://doi.org/10.1002/adma.200500863.

(39) Limo, M. J.; Ramasamy, R.; Perry, C. C. ZnO Binding Peptides: Smart Versatile Tools for Controlled Modification of ZnO Growth Mechanism and Morphology. Chem. Mater. 2015, 27 (6), 1950-1960. https://doi.org/10.1021/acs.chemmater.5b00419.

(40) Sola-Rabada, A.; Liang, M.-K.; Roe, M. J.; Perry, C. C. Peptide-Directed Crystal Growth Modification in the Formation of ZnO. J. Mater. Chem. B 2015, 3 (18), 3777-3788. https://doi.org/10.1039/C5TB00253B. 
(41) Baier, J.; Blumenstein, N. J.; Preusker, J.; Jeurgens, L. P. H.; Welzel, U.; Do, T. A.; Pleiss, J.; Bill, J. The Influence of ZnO-Binding 12-Mer Peptides on Bio-Inspired ZnO Formation. CrystEngComm 2014, 16 (24), 5301. https://doi.org/10.1039/c4ce00520a.

(42) Liang, M.-K.; Limo, M. J.; Sola-Rabada, A.; Roe, M. J.; Perry, C. C. New Insights into the Mechanism of ZnO Formation from Aqueous Solutions of Zinc Acetate and Zinc Nitrate. Chem. Mater. 2014, 26 (14), 4119-4129. https://doi.org/10.1021/cm501096p.

(43) Liang, M.-K.; Deschaume, O.; Patwardhan, S. V; Perry, C. C. Direct Evidence of ZnO Morphology Modification via the Selective Adsorption of ZnO-Binding Peptides. J. Mater. Chem. 2011, 21 (1), 80-89. https://doi.org/10.1039/C0JM02124E.

(44) Rothenstein, D.; Claasen, B.; Omiecienski, B.; Lammel, P.; Bill, J. Isolation of ZnOBinding 12-Mer Peptides and Determination of Their Binding Epitopes by NMR Spectroscopy. J. Am. Chem. Soc. 2012, 134 (30), 12547-12556. https://doi.org/10.1021/ja302211w.

(45) Dudev, T.; Lim, C. Principles Governing Mg, Ca, and Zn Binding and Selectivity in Proteins. Chem. Rev. 2003, 103 (3), 773-788. https://doi.org/10.1021/cr020467n.

(46) Maret, W.; Li, Y. Coordination Dynamics of Zinc in Proteins. Chem. Rev. 2009, 109 (10), 4682-4707. https://doi.org/10.1021/cr800556u.

(47) Vallee, B. L.; Auld, D. S. Active-Site Zinc Ligands and Activated H2O of Zinc Enzymes. Proc. Natl. Acad. Sci. 1990, 87 (1), 220-224. https://doi.org/10.1073/pnas.87.1.220.

(48) Laitaoja, M.; Valjakka, J.; Jänis, J. Zinc Coordination Spheres in Protein Structures. Inorg. 
Chem. 2013, 52 (19), 10983-10991. https://doi.org/10.1021/ic401072d.

(49) Kay, B. K.; Adey, N. B.; Yun-Sheng, H.; Manfredi, J. P.; Mataragnon, A. H.; Fowlkes, D. M. An M13 Phage Library Displaying Random 38-Amino-Acid Peptides as a Source of Novel Sequences with Affinity to Selected Targets. Gene 1993, 128 (1), 59-65. https://doi.org/10.1016/0378-1119(93)90153-T.

(50) Peters, E. A.; Schatz, P. J.; Johnson, S. S.; Dower, W. J. Membrane Insertion Defects Caused by Positive Charges in the Early Mature Region of Protein PIII of Filamentous Phage Fd Can Be Corrected by PrlA Suppressors. J. Bacteriol. 1994, 176 (14), 4296-4305. https://doi.org/10.1128/jb.176.14.4296-4305.1994.

(51) Rai, A.; Perry, C. C. Facile Fabrication of Uniform Silica Films with Tunable Physical Properties Using Silicatein Protein from Sponges. Langmuir 2010, 26 (6), 4152-4159. https://doi.org/10.1021/la903366a.

(52) Hickman, G. J.; Rai, A.; Boocock, D. J.; Rees, R. C.; Perry, C. C. Fabrication, Characterisation and Performance of Hydrophilic and Super-Hydrophilic Silica as Cell Culture Surfaces. J. Mater. Chem. 2012, 22 (24), 12141-12148. https://doi.org/10.1039/c2jm31161e.

(53) Nicklin, M.; Rees, R. C.; Pockley, A. G.; Perry, C. C. Development of an Hydrophobic Fluoro-Silica Surface for Studying Homotypic Cancer Cell Aggregation-Disaggregation as a Single Dynamic Process in Vitro. Biomater. Sci. 2014, 2 (10), 1486-1496. https://doi.org/10.1039/C4BM00194J.

(54) Michaelis, M.; Fischer, C.; Colombi Ciacchi, L.; Luttge, A. Variability of Zinc Oxide 
Dissolution Rates. Environ. Sci. Technol. 2017, 51 (8), 4297-4305. https://doi.org/10.1021/acs.est.6b05732.

(55) Diwu, Z.; Chen, C.-S.; Zhang, C.; Klaubert, D. H.; Haugland, R. P. A Novel Acidotropic PH Indicator and Its Potential Application in Labeling Acidic Organelles of Live Cells. Chem. Biol. 1999, 6 (7), 411-418. https://doi.org/https://doi.org/10.1016/S10745521(99)80059-3.

(56) Gee, K. .; Zhou, Z.-L.; Ton-That, D.; Sensi, S. .; Weiss, J. . Measuring Zinc in Living Cells. Cell Calcium 2002, 31 (5), 245-251. https://doi.org/10.1016/S0143-4160(02)00053-2.

(57) Jiang, P.; Guo, Z. Fluorescent Detection of Zinc in Biological Systems: Recent Development on the Design of Chemosensors and Biosensors. Coord. Chem. Rev. 2004, 248 (1-2), 205-229. https://doi.org/10.1016/j.cct.2003.10.013.

(58) Yung-Chi, C.; Prusoff, W. H. Relationship between the Inhibition Constant (KI) and the Concentration of Inhibitor Which Causes 50 per Cent Inhibition (I50) of an Enzymatic Reaction. Biochem. Pharmacol. 1973, 22 (23), 3099-3108. https://doi.org/10.1016/00062952(73)90196-2.

(59) Craig, D. A. The Cheng-Prusoff Relationship: Something Lost in the Translation. Trends Pharmacol. Sci. 1993, 14 (3), 89-91. https://doi.org/10.1016/0165-6147(93)90070-Z.

(60) Lazareno, S.; Birdsall, N. J. M. Estimation of Competitive Antagonist Affinity from Functional Inhibition Curves Using the Gaddum, Schild and Cheng-Prusoíf Equations. Br. J. Pharmacol. 1993, 109 (4), 1110-1119. https://doi.org/10.1111/j.14765381.1993.tb13737.x. 
(61) Cheng, H. C. The Power Issue: Determination of KB or Ki from IC50. J. Pharmacol. Toxicol. Methods 2001, 46 (2), 61-71. https://doi.org/10.1016/S1056-8719(02)00166-1.

(62) Cheng, H. The Influence of Cooperativity on the Determination of Dissociation Constants: Examination of the Cheng-Prusoff Equation, the Scatchard Analysis, the Schild Analysis and Related Power Equations. Pharmacol. Res. 2004, 50 (1), 21-40. https://doi.org/10.1016/j.phrs.2003.11.007.

(63) Alsteens, D.; Pfreundschuh, M.; Zhang, C.; Spoerri, P. M.; Coughlin, S. R.; Kobilka, B. K.; Müller, D. J. Imaging G Protein-Coupled Receptors While Quantifying Their LigandBinding Free-Energy Landscape. Nat. Methods 2015, 12 (9), 845-851. https://doi.org/10.1038/nmeth.3479.

(64) Kilper, S.; Facey, S. J.; Burghard, Z.; Hauer, B.; Rothenstein, D.; Bill, J. Macroscopic Properties of Biomimetic Ceramics Are Governed by the Molecular Recognition at the Bioorganic-Inorganic Interface. Adv. Funct. Mater. 2018, 28 (10), 1705842. https://doi.org/10.1002/adfm.201705842.

(65) Kilper, S.; Jahnke, T.; Aulich, M.; Burghard, Z.; Rothenstein, D.; Bill, J. Genetically Induced In Situ-Poling for Piezo-Active Biohybrid Nanowires. Adv. Mater. 2019, 31 (6), 1805597. https://doi.org/10.1002/adma.201805597.

(66) Belton, D.; Paine, G.; Patwardhan, S. V; Perry, C. C. Towards an Understanding of (Bio)Silicification: The Role of Amino Acids and Lysine Oligomers in Silicification. $J$. Mater. Chem. 2004, 14 (14), 2231-2241. https://doi.org/10.1039/B401882F.

(67) Mathé, C.; Devineau, S.; Aude, J.-C.; Lagniel, G.; Chédin, S.; Legros, V.; Mathon, M.-H.; 
Renault, J.-P.; Pin, S.; Boulard, Y.; et al. Structural Determinants for Protein Adsorption/Non-Adsorption to Silica Surface. PLoS One 2013, 8 (11), e81346. https://doi.org/10.1371/journal.pone.0081346.

(68) Otzen, D. The Role of Proteins in Biosilicification. Scientifica (Cairo). 2012, 2012 (Article ID 867562). https://doi.org/10.6064/2012/867562.

(69) Belton, D. J.; Patwardhan, S. V; Perry, C. C. Spermine, Spermidine and Their Analogues Generate Tailored Silicas. J. Mater. Chem. 2005, 15 (43), 4629-4638. https://doi.org/10.1039/B509683A.

(70) Belton, D. J.; Patwardhan, S. V; Annenkov, V. V; Danilovtseva, E. N.; Perry, C. C. From Biosilicification to Tailored Materials: Optimizing Hydrophobic Domains and Resistance to Protonation of Polyamines. Proc. Natl. Acad. Sci. 2008, 105 (16), 5963-5968. https://doi.org/10.1073/pnas.0710809105.

(71) Rimola, A.; Sodupe, M.; Ugliengo, P. Affinity Scale for the Interaction of Amino Acids with Silica Surfaces. J. Phys. Chem. C 2009, 113 (14), 5741-5750. https://doi.org/10.1021/jp811193f.

(72) Patwardhan, S. V; Emami, F. S.; Berry, R. J.; Jones, S. E.; Naik, R. R.; Deschaume, O.; Heinz, H.; Perry, C. C. Chemistry of Aqueous Silica Nanoparticle Surfaces and the Mechanism of Selective Peptide Adsorption. J. Am. Chem. Soc. 2012, 134 (14), 6244-6256. https://doi.org/10.1021/ja211307u.

(73) Emami, F. S.; Puddu, V.; Berry, R. J.; Varshney, V.; Patwardhan, S. V; Perry, C. C.; Heinz, H. Prediction of Specific Biomolecule Adsorption on Silica Surfaces as a Function of PH 
and Particle Size. Chem. Mater. 2014, 26 (19), 5725-5734. https://doi.org/10.1021/cm5026987.

(74) Han, J. W.; Sholl, D. S. Enantiospecific Adsorption of Amino Acids on Hydroxylated Quartz (0001). Langmuir 2009, 25 (18), 10737-10745. https://doi.org/10.1021/la901264e.

(75) Han, J. W.; Sholl, D. S. Enantiospecific Adsorption of Amino Acids on Hydroxylated Quartz (100). Phys. Chem. Chem. Phys. 2010, 12 (28), 8024-8032. https://doi.org/10.1039/B926035H.

(76) Wright, L. B.; Walsh, T. R. Facet Selectivity of Binding on Quartz Surfaces: Free Energy Calculations of Amino-Acid Analogue Adsorption. J. Phys. Chem. C 2012, 116 (4), 29332945. https://doi.org/10.1021/jp209554g.

(77) Menzel, H.; Horstmann, S.; Behrens, P.; Bärnreuther, P.; Krueger, I.; Jahns, M. Chemical Properties of Polyamines with Relevance to the Biomineralization of Silica. Chem. Commun. 2003, No. 24, 2994-2995. https://doi.org/10.1039/B310201G.

(78) Delle Piane, M.; Vaccari, S.; Corno, M.; Ugliengo, P. Silica-Based Materials as Drug Adsorbents: First Principle Investigation on the Role of Water Microsolvation on Ibuprofen Adsorption. J. Phys. Chem. A 2014, 118 (31), 5801-5807. https://doi.org/10.1021/jp411173k.

(79) Zhou, L.; Li, S.; Su, Y.; Yi, X.; Zheng, A.; Deng, F. Interaction between Histidine and Zn(II) Metal Ions over a Wide PH as Revealed by Solid-State NMR Spectroscopy and DFT $\begin{array}{llllllll}\text { Calculations. J. } & \text { Phys. Chem. } & \text { B 2013, } 117 & \text { (30), 8954-8965. }\end{array}$ https://doi.org/10.1021/jp4041937. 
(80) Trzaskowski, B.; Adamowicz, L.; Deymier, P. A. A Theoretical Study of Zinc(II) Interactions with Amino Acid Models and Peptide Fragments. JBIC J. Biol. Inorg. Chem. 2007, 13 (1), 133-137. https://doi.org/10.1007/s00775-007-0306-y.

(81) Liao, S.-M.; Du, Q.-S.; Meng, J.-Z.; Pang, Z.-W.; Huang, R.-B. The Multiple Roles of Histidine in Protein Interactions. Chem. Cent. J. 2013, 7 (1), 44. https://doi.org/10.1186/1752-153X-7-44.

(82) Chakrabarti, P. Geometry of Interaction of Metal Ions with Histidine Residues in Protein Structures. $\quad$ "Protein Eng. Des. Sel. 1990, 4 (1), 57-63. https://doi.org/10.1093/protein/4.1.57.

(83) Lin; Lim, C. Factors Governing the Protonation State of Zn-Bound Histidine in Proteins: A DFT/CDM Study. J. Am. Chem. Soc. 2004, 126 (8), 2602-2612. https://doi.org/10.1021/ja038827r.

(84) Daniel, A. G.; Farrell, N. P. The Dynamics of Zinc Sites in Proteins: Electronic Basis for Coordination Sphere Expansion at Structural Sites. Metallomics 2014, 6 (12), 2230-2241. https://doi.org/10.1039/C4MT00213J.

(85) Gerstel, P.; Hoffmann, R. C.; Lipowsky, P.; Jeurgens, L. P. H.; Bill, J.; Aldinger, F. Mineralization from Aqueous Solutions of Zinc Salts Directed by Amino Acids and Peptides. Chem. Mater. 2006, 18 (1), 179-186. https://doi.org/10.1021/cm051542o.

(86) Gerstel, P.; Lipowsky, P.; Durupthy, O.; Hoffmann, R. C.; Bellina, P.; Bill, J.; Aldinger, F. Deposition of Zinc Oxide and Layered Basic Zinc Salts from Aqueous Solutions Containing Amino Acids and Dipeptides. J. Ceram. Soc. Japan 2006, 114 (1335), 911-917. 
https://doi.org/10.2109/jcersj.114.911.

(87) große Holthaus, S.; Köppen, S.; Frauenheim, T.; Ciacchi, L. C. Molecular Dynamics Simulations of the Amino Acid-ZnO (10-10) Interface: A Comparison between Density Functional Theory and Density Functional Tight Binding Results. J. Chem. Phys. 2014, 140 (23), 234707. https://doi.org/10.1063/1.4882280.

(88) Gao, Y. K.; Traeger, F.; Shekhah, O.; Idriss, H.; Wöll, C. Probing the Interaction of the Amino Acid Alanine with the Surface of ZnO. J. Colloid Interface Sci. 2009, 338 (1), 1621. https://doi.org/10.1016/j.jcis.2009.06.008.

(89) Hill, A. V. The Possible Effects of the Aggregation of the Molecules of Haemoglobin on Its Dissociation Curves. J Physiol 1910, 40, 4-7.

(90) Clark, A. J. Mode of Action of Drugs on Cells; Edward Arnold: London, 1933.

(91) Ruffolo, R. R. Important Concepts of Receptor Theory. J. Auton. Pharmacol. 1982, 2 (4), 277-295. https://doi.org/10.1111/j.1474-8673.1982.tb00520.x.

(92) Colquhoun, D. The Quantitative Analysis of Drug-Receptor Interactions: A Short History. Trends Pharmacol. Sci. 2006, 27 (3), 149-157. https://doi.org/10.1016/j.tips.2006.01.008. 
TOC

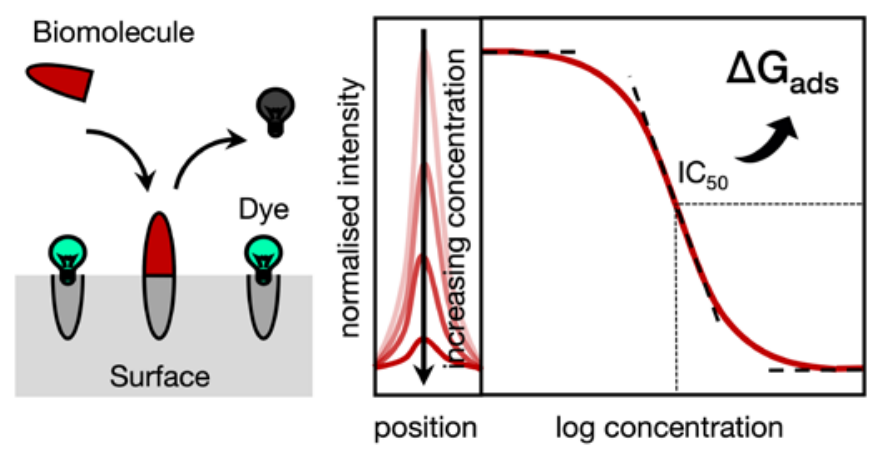


